\title{
Injuria encefálica aguda en el posoperatorio de cirugía cardíaca
}

\author{
Dres. Mauricio Sáez, Omar Montes De Oca
}

\section{Resumen}

La injuria encefálica aguda es una de las complicaciones más devastadoras en el posoperatorio de cirugía cardíaca, siendo responsable de un mayor tiempo de asistencia respiratoria mecánica, infecciones pulmonares y por catéteres, dehiscencia esternal, mayor tiempo de estadía hospitalaria, aumento de mortalidad y de costos sanitarios. En el presente trabajo se discuten las diferentes formas de presentación, los factores predisponentes, su fisiopatología, las medidas de prevención y el tratamiento.

Palabras clave: CIRUGÍA CARDÍACA

POSOPERATORIO

COMPLICACIONES NEUROLÓGICAS

ATAQUE CEREBROVASCULAR

DELIRIO

\section{Acute brain injury in the postoperative period of cardiac surgery \\ Summary}

Acute brain injury is one of the most devastating complications in the postoperative period of cardiac surgery, being responsible for a longer time of mechanical ventilation, lung and catheter infections, sternal dehiscence, longer hospital stay, increased mortality and healthcare costs. The present work discusses the different forms of presentation, predisposing factors, their pathophysiology, prevention measures and treatment.

Key words: $\quad$ CARDIAC SURGERY

POSTOPERATIVE

NEUROLOGICAL COMPLICATIONS

STROKE

DELIRIUM

\section{Lesão encefálica aguda no pós-operatório de cirurgia cardíaca}

\section{Resumo}

O dano cerebral agudo é uma das complicações mais devastadoras no pós-operatório de cirurgia cardíaca, sendo responsável por maior tempo de ventilação mecânica, infecções pulmonares, infecções de cateter, deiscência esternal, maior tempo de internação, aumento da mortalidade e custos de saúde. O presente trabalho discute as diferentes formas de apresentação, fatores predisponentes, sua fisiopatologia, medidas de prevenção e tratamento.

Palavras chave: CIRURGIA CARDÍACA

PÓS-OPERATÓRIO

COMPLICAÇOES NEUROLÓGICAS

ACIDENTE VASCULAR CEREBRAL

DELÍRIO

Centro de Investigación Cardiovascular Uruguayo (CICU), Casa de Galicia. Montevideo, Uruguay.

Correspondencia: Dr. Mauricio Sáez. Correo electrónico: masaezrepetto@gmail.com

Los autores declaran no tener conflictos de intereses.

Recibido Oct 28, 2020; aceptado Nov 6, 2020 


\section{Introducción}

La Injuria encefálica aguda (IEA), sigue siendo una complicación importante y a veces devastadora en el posoperatorio de cirugía cardíaca (POCC). Una complicación neurológica puede definirse como cualquier lesión temporal o permanente del sistema nervioso central y/o periférico, pudiendo comprometer hemisferios cerebrales, médula espinal y nervios periféricos ${ }^{(1)}$. La IEA se manifiesta como ataque cerebrovascular (stroke) y/o trastornos neurocognitivos, y es responsable de un mayor tiempo de asistencia respiratoria mecánica, infecciones bronco-pulmonares, infecciones por catéteres, dehiscencias esternales mecánicas, mayor tiempo de estadía hospitalaria, aumento de mortalidad y costos más elevados. Según las diferentes estadísticas, la incidencia de stroke oscila entre $0,8 \%-5,2 \%$, y los trastornos cognitivos entre 50\%-80\%. El stroke es una de las principales causas de morbimortalidad y readmisión en el POCC, con una mortalidad aproximada de $12 \%(2,28)$. Lamentablemente, aún no se ha desarrollado una neuroprotección ideal ${ }^{(3)}$.

\section{Factores predisponentes}

Los principales predisponentes de IEA en el POCC son la edad, historia de enfermedad cerebrovascular, diabetes e hipertensión arterial. La población sometida a cirugía cardíaca en la actualidad ha cambiado con respecto a décadas anteriores, debido a las características de los pacientes, destacándose una edad mayor y un número elevado de comorbilidades, al tiempo de constatarse procedimientos quirúrgicos más complejos. Existen predictores vinculados a factores preoperatorios, intraoperatorios y posoperatorios, que se detallan en la_tabla $1^{(10,18)}$.

\section{Clasificación}

El American College of Cardiology (ACC) y la American Heart Association (AHA) clasifican las IEA en:

- Tipo I:

- Ataque isquémico transitorio (AIT) (stroke).

- Estupor.

- Coma.

- Tipo II:

- Convulsiones.

- Afectación de la función intelectual y memoria.

- Trastorno cognitivo.
Fisiopatología

Estas alteraciones encefálicas presentadas en el $\mathrm{POCC}$ son de causa multifactorial, siendo la isquemia focal o global la principal involucrada. Las tres etiologías más frecuentes son:

1. Embolización (macro o micro embolización).

2. Hipoperfusión cerebral.

3. Respuesta inflamatoria.

La embolización e hipoperfusión explican la mayor parte de las IEA, la macroembolización es causa de stroke en más del $60 \%$ de los casos, mientras que las alteraciones neurocognitivas están vinculadas principalmente a microembolización. El material embolizado puede ser aire (mayor en cirugía cardíaca con apertura de cavidades) así como material aterotrombótico (vinculado a manipulación aórtica), o cálcico, colesterínico o fibrinoplaquetario.

La hipoperfusión cerebral es otra de las causas en las alteraciones neuropsíquicas. Habitualmente el flujo sanguíneo cerebral (FSC) normal es de 60 $\mathrm{ml} / 100 \mathrm{~g}$ de tejido cerebral/min. Uno de los principales reguladores del FSC es la presión arterial media (PAM), y el descenso de la misma trae asociada isquemia encefálica. Normalmente la autorregulación cerebral varía entre $50-150 \mathrm{mmHg}$, las PAM alejadas de dichos valores pueden traer aparejados isquemia encefálica o edema vasogénico ${ }^{(7)}$. Se reconocen como factores predictores de pérdida de la autorregulación la edad, la hipertensión arterial y la diabetes.

Otra causa de IEA es la respuesta inflamatoria generada durante el procedimiento de cirugía cardíaca. Su incidencia es mayor en pacientes sometidos a circulación extracorpórea (CEC) aunque también puede estar presente en pacientes intervenidos sin CEC. No se han constatado diferencias significativas en la IEA en cirugía de revascularización miocárdica con CEC o sin $\mathrm{CEC}^{(1,3)}$.

Durante la cirugía cardíaca se liberan múltiples mediadores, siendo las citoquinas las más frecuentes, las cuales aumentan en plasma y en el líquido cefalorraquídeo de los pacientes ${ }^{(5,6)}$. El contacto prolongado de la sangre con materiales ajenos al organismo tales como los circuitos de CEC y la isquemia-reperfusión generan mecanismos inflamatorios e inmunológicos cuya consecuencia son las alteraciones de la permeabilidad y de estructuras celulares. Para minimizar la IEA se debe realiza hipotermia moderada $\left(20-28^{\circ} \mathrm{C}\right)$ seguida de recalentamiento lento y progresivo para prevenir las lesiones isquémicas durante la reperfusión.

La estenosis carotídea ha sido identificada como factor de riesgo en la instalación de stroke periope- 


\begin{tabular}{|c|c|c|}
\hline \multicolumn{3}{|c|}{ a) Cirugía de revascularización miocárdica con circulación extracorpórea } \\
\hline Preoperatorio & Intraoperatorio & Posoperatorio \\
\hline Edad avanzada & Cirugía de urgencia & Fibrilación auricular \\
\hline Sexo femenino & $\begin{array}{l}\text { Manipulación de aorta ascendente } \\
\text { ateromatosa }\end{array}$ & Síndrome de bajo gasto \\
\hline Stroke o AIT previo & Tiempo de CEC $>2 \mathrm{~h}$ & \\
\hline Enfermedad de pequeño vaso & Clampeo aórtico intermitente & \\
\hline Ateromatosis carotídea & Hipotensión arterial severa & \\
\hline Ateromatosis aórtica proximal & Bajo nivel de hematocrito & \\
\hline Enfermedad arterial periférica & Necesidad de hemofiltración & \\
\hline Cirugía cardíaca previa & Politransfusión & \\
\hline \multicolumn{3}{|l|}{ Infarto de miocardio reciente } \\
\hline \multicolumn{3}{|l|}{ Disfunción ventricular izquierda } \\
\hline \multicolumn{3}{|l|}{ Trombo intracardíaco } \\
\hline \multicolumn{3}{|l|}{ Diabetes } \\
\hline \multicolumn{3}{|l|}{ Presión del pulso elevada } \\
\hline \multicolumn{3}{|l|}{ Hipertensión arterial } \\
\hline \multicolumn{3}{|l|}{ Tabaquismo } \\
\hline \multicolumn{3}{|l|}{ Enfermedad renal crónica } \\
\hline \multicolumn{3}{|l|}{ Infección preoperatoria } \\
\hline \multicolumn{3}{|c|}{ b) Paro circulatorio con hipotermia profunda } \\
\hline \multicolumn{3}{|l|}{ Cirugía de urgencia } \\
\hline \multicolumn{3}{|l|}{ Duración de la CEC } \\
\hline \multicolumn{3}{|c|}{ Duración del paro circulatorio hipotérmico } \\
\hline
\end{tabular}

AIT: ataque isquémico transitorio; CEC: circulación extracorpórea. Modificado de ${ }^{(10)} \mathrm{y}^{(18)}$.

ratorio, favoreciendo complicaciones embólicas, hipoperfusión y deterioro de la autorregulación distal a la estenosis u oclusión de la lesión carotídea. Las arritmias cardíacas son una complicación frecuente en el POCC, siendo la fibrilación auricular (FA) la de mayor incidencia (20\%-40\% de los POCC según diferentes series publicadas). La mayoría de los pacientes instalan FA habitualmente en los primeros tres días del POCC, la cual es causa de complicaciones cardioembólicas. La relación entre FA y stroke ha sido documentada por múltiples estudios, y es la causa en más de un tercio de los pacientes con stroke isquémico. La FA en el POCC es un predictor independiente de IEA.

\section{Diagnóstico y presentación del stroke}

Se define stroke posoperatorio como un cuadro de instalación brusca de un nuevo déficit neurológico, focal o global, en los primeros 30 días del POCC como consecuencia de una alteración de origen vascular, de duración mayor de $24 \mathrm{~h}^{(31)}$ y con evidencia imagenológica de lesión estructural. Afecta más frecuentemente la circulación cerebral anterior. El AIT es el compromiso neurológico manifestado habitualmente por trastornos simbólicos o focales de menos de $24 \mathrm{~h}$ de duración sin evidencia imagenológica de lesión estructural encefálica aguda. La incidencia de stroke en el POCC oscila según las series publicadas, siendo casi del 6\%. La mayor incidencia 


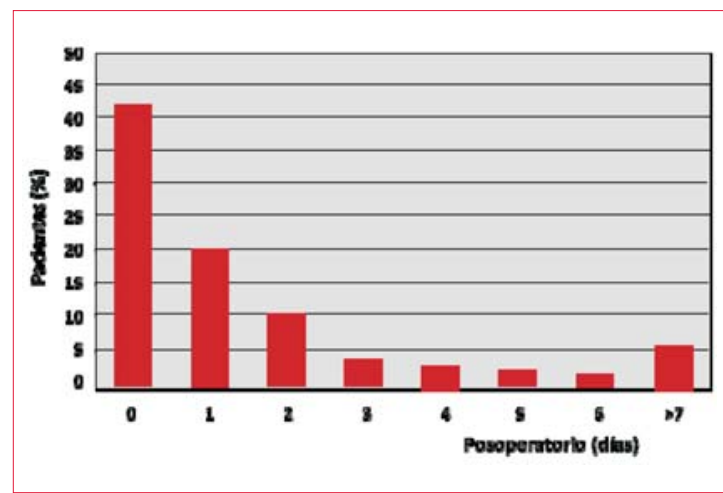

Figura 1. Frecuencia de stroke según el momento de posoperatorio. Modificado de ${ }^{(8)}$

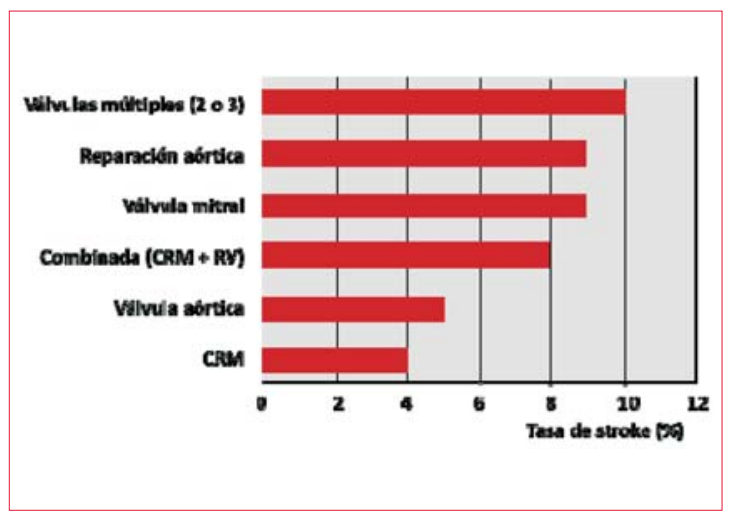

Figura 3. Frecuencia de stroke según procedimiento realizado. CRM: cirugía de revascularización miocárdica; RV: reparación valvular. Modificado de ${ }^{(9)}$

se observa en los primeros dos días del POCC ( $42 \%$ en el primer día, 20\% en el segundo día) (figura 1). La edad es un predictor mayor, teniendo una relación directamente proporcional en pacientes mayores de 80 años (figura 2).

La IEA se relaciona también con el procedimiento quirúrgico realizado y aumenta con su complejidad, siendo menor el riesgo en cirugía de revascularización miocárdica (CRM) y mayor en cirugía combinada: CRM más sustitución valvular (figura 3). Asimismo, la realización de un procedimiento vascular periférico simultáneo, como endarterectomía carotídea, incrementa la morbimortalidad ${ }^{(9,10)}$.

El diagnóstico de stroke es clínico e imagenológico. La tomografía de cráneo (TAC) precoz habitualmente no evidencia lesiones estructurales. La resonancia nuclear magnética (RNM) es también una herramienta útil para certificar el diagnóstico, naturaleza del cuadro, topografía, extensión y complicaciones asociadas. El electroencefalograma (EEG) forma parte del screening diagnóstico para evidenciar sufrimiento encefálico y actividad epileptógena. El eco-Doppler color transcraneano (EDCTC) es de excelente utilidad en la evaluación del paciente

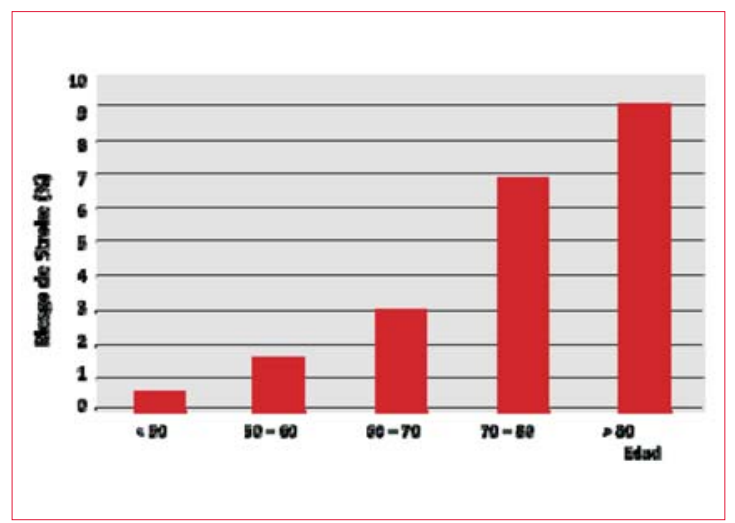

Figura 2. Frecuencia de stroke según la edad. Modificado de ${ }^{(9)}$

con stroke isquémico o hemorrágico porque además de evaluar el FSC en tiempo real nos permite observar la anatomía cerebral. En la actualidad existe una amplia experiencia con el EDCTC tanto en la valoración de la circulación cerebral como en la monitorización de la recanalización con acción terapéutica asociada (son-trombólisis), detección de microembolias e incluso la detección de cortocircuito derecha-izquierda ${ }^{(40)}$. El paciente cursando un POCC es un paciente crítico, muchas veces en asistencia respiratoria mecánica (ARM), inestable, con apoyo de drogas inotrópicas y vasopresoras, por lo que su traslado para estudio imagenológico puede favorecer complicaciones secundarias a hipoxemia/hipoperfusión, y debe evaluarse el riesgo/beneficio para definir su oportunidad y la conducta ulterior.

\section{Diagnóstico y presentación del delirio}

El delirio, otra de las alteraciones neurocognitivas presentes en el POCC, es la alteración en el nivel de conciencia y atención acompañada de disfunciones cognitivas, pudiendo asociar cambios emocionales, autonómicos o conductuales de instauración más o menos aguda, progresiva y fluctuante. El delirio puede clasificarse en hipoactivo, hiperactivo y mixto; el hipoactivo se caracteriza por somnolencia, decaimiento y bradipsiquia (peor pronóstico); el hiperactivo o agresivo se manifiesta por un estado hiperalerta con alucinaciones y agitación psicomotriz ${ }^{(11-13)}$. La causa del delirio es multifactorial, y existe una interrelación entre la vulnerabilidad del paciente y factores predisponentes, como edad avanzada, consumo de alcohol o psicofármacos, y exposición a factores precipitantes como hospitalización, enfermedad aguda, inmovilidad, implantes de catéteres (sonda nasogástrica, sonda vesical, catéteres venosos centrales, catéteres arteriales), dolor, hipoxia y alteraciones metabólicas (figura 4). 


\begin{tabular}{|c|c|c|c|}
\hline & Preoperatorio & Intraoperatorio & Posoperatorio \\
\hline \multirow[t]{6}{*}{ Modificable } & $\begin{array}{l}\text { Control de } \\
\text { presión arterial }\end{array}$ & $\begin{array}{l}\text { Manipulación } \\
\text { quirúrgica }\end{array}$ & $\begin{array}{l}\text { Duración de la ventilación } \\
\text { mecánica }\end{array}$ \\
\hline & Control glucémico & $\begin{array}{l}\text { Derivación } \\
\text { cardiopulmonar }\end{array}$ & $\begin{array}{l}\text { Sedación, analgesia y } \\
\text { manejo del delirio }\end{array}$ \\
\hline & \multirow{4}{*}{$\begin{array}{l}\text { Abuso de alcohol, nicotina } \\
\text { o sustancias }\end{array}$} & Gestión de la temperatura & \\
\hline & & Manejo del hematocrito & \\
\hline & & Presión arterial & \\
\hline & & Control glucémico & \\
\hline \multirow[t]{4}{*}{ Parcialmente modificable } & Reserva neurocognitiva & $\begin{array}{l}\text { Manejo anestésico y } \\
\text { control del índice } \\
\text { biespectral }\end{array}$ & $\begin{array}{l}\text { Complicaciones } \\
\text { posoperatorias }\end{array}$ \\
\hline & Depresión y ansiedad & $\begin{array}{l}\text { Control cerebral de } \\
\text { oxígeno }\end{array}$ & Fragilidad del paciente \\
\hline & Nivel educativo & \multirow[t]{2}{*}{ Duración de la cirugía } & \multirow[t]{2}{*}{ Trastornos del sueño } \\
\hline & Ajuste social & & \\
\hline No modificable & $\begin{array}{l}\text { Edad } \\
\text { Sexo femenino } \\
\text { Genética } \\
\text { Demencia } \\
\text { Enfermedad } \\
\text { neurodegenerativa } \\
\text { Enfermedad vascular } \\
\text { subyacente } \\
\text { Enfermedad renal y } \\
\text { hepática } \\
\text { Enfermedad pulmonar }\end{array}$ & Tipo de cirugía & Ambiente hospitalario \\
\hline
\end{tabular}

El delirio está relacionado con estadías más prolongadas en la unidad de cuidados intensivos (UCI), mayor período de ARM, mayor incidencia de infección, aumento de mortalidad y de costos.

La mayoría de los pacientes que presentan delirio lo instalan en las primeras $48 \mathrm{~h}$ del POCC y la duración es habitualmente hasta un año de la cirugía pero puede mantenerse por periodos más prologa$\operatorname{dos}^{(17,18)}$.

\section{Prevención y tratamiento}

El mayor efecto neuroprotector está dado por el mantenimiento de las variables fisiológicas mayores, con una adecuada perfusión y oxigenación cerebral en el pre, intra y POCC. Es importante mantener buena hemodinamia, con valores de PAM $\geq 70$ $\mathrm{mmHg}$, evitar la hiperglicemia, controlar alteraciones hidroelectrolíticas y del equilibrio ácido-base, mantener una saturación arterial de oxígeno
$>95 \%$. Los esfuerzos para la prevención deben ser realizados a diferentes niveles.

\section{Prevención prequirúrgica}

En pacientes con cardiopatía isquémica hay una alta prevalencia de enfermedad carotídea.

Pacientes con lesión severa de tronco de arteria coronaria izquierda (TCI) por cineangiocoronariografía asocian lesiones carotídeas significativas en un $25 \%$ de los casos. Se debe evaluar con Doppler carotídeo $^{(35)}$ a aquellos pacientes con:

1. Edad mayor de 65 años.

2. Lesión severa de TCI.

3. Portadores de enfermedad vascular periférica.

4. Antecedentes de enfermedad cerebrovascular (AIT - stroke).

5. Hipertensos.

6. Fumadores.

7. Diabéticos. 


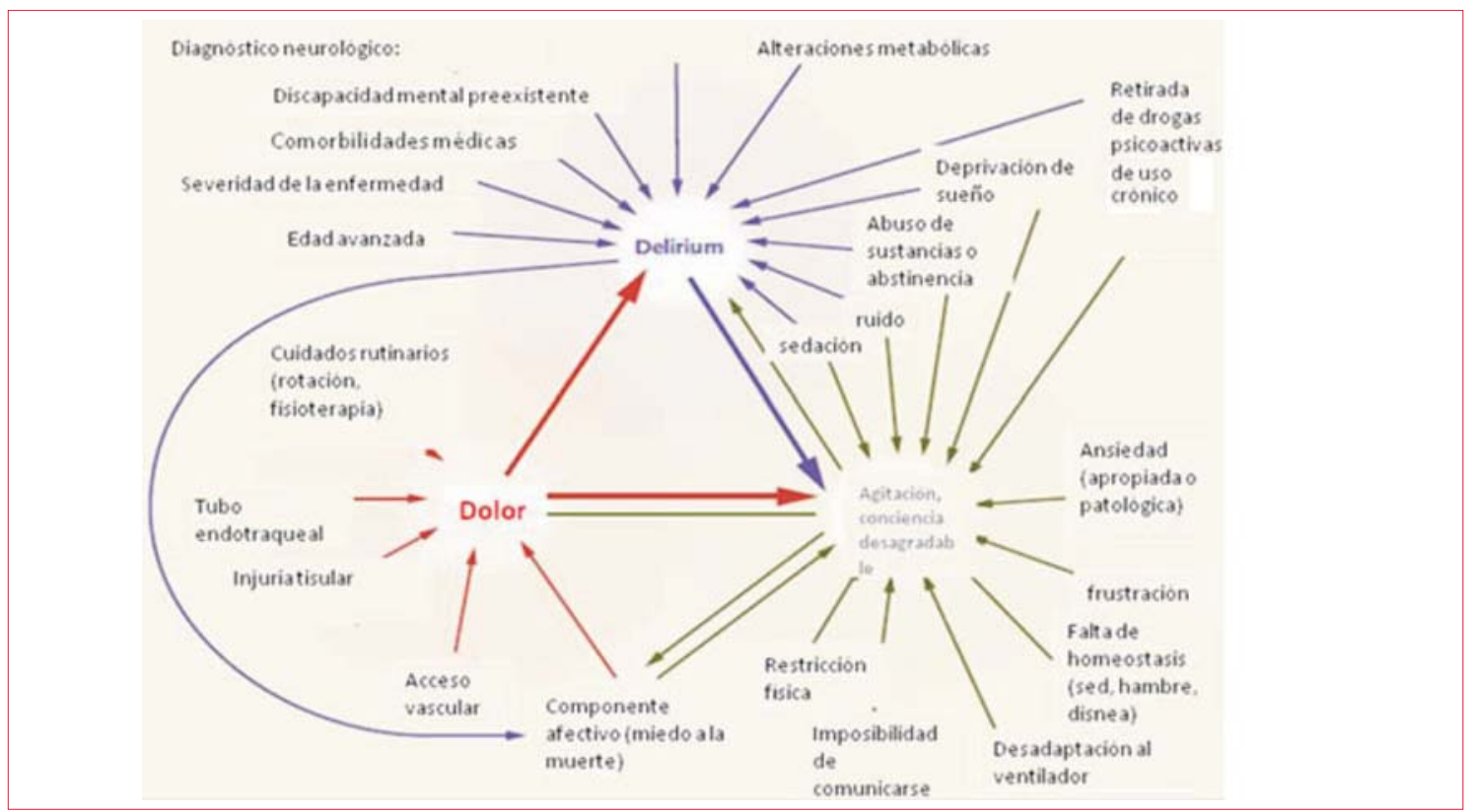

Figura 4. Factores relacionados con el delirio en el posoperatorio(15).

De acuerdo a los síntomas, severidad de las lesiones y topografía, se planificará endarterectomía carotídea previa o simultánea con la intervención cardíaca.

La realización del ecocardiograma transesofágico intraoperatorio está justificada para evidenciar placas de ateroma y su localización en la aorta, para poder definir el sitio correcto donde realizar el clampeo aórtico o canulación arterial.

De acuerdo al tiempo que medie entre la evaluación pre-quirúrgica y realización de la cirugía es conveniente lograr una adecuada psico-profilaxis para minimizar la ansiedad y el estrés perioperatorio, explicando al paciente el procedimiento, el posoperatorio, el tiempo usual de internación y retorno a su vida habitual.

Es relevante lograr un adecuado control metabólico, especialmente en diabéticos y en caso de trastornos hidroelectrolíticos, optimizar el tratamiento antihipertensivo y antianginoso, lograr estabilización hemodinámica y prevenir arritmias.

\section{Prevención intraquirúrgica}

La mayor parte de las embolias que causan stroke en el POCC provienen de placas ateromatosas de la aorta ascendente, siendo su presencia un factor de riesgo independiente de stroke durante la cirugía cardíaca. La presencia de placas de ateroma en el arco aórtico cuadriplica la incidencia de stroke, que se caracteriza por lesiones isquémicas múltiples en diferentes territorios vasculares. Independientemente del procedimiento quirúrgico, los pacientes con placas de ateroma mayores de $4 \mathrm{~mm}$ tienen una incidencia anual de stroke recurrente del $12 \%(41)$.

Los circuitos de CEC están diseñados para reducir complicaciones embólicas y el trauma de los componentes sanguíneos, proporcionan un flujo sanguíneo fisiológico preservando el funcionamiento plaquetario, evitando activación de procoagulantes, fibrinólisis, el sangrado y necesidad de transfusiones. Sin embargo, la CEC no solo es fuente de embolias sino también un poderoso estímulo para la activación de la respuesta inflamatoria sistémica. La sangre en contacto con superficies del circuito genera daño por isquemia-reperfusión, activando el complemento y la liberación de citoquinas proinflamatorias que estimulan leucocitos y células endoteliales capaces de sensibilizar la vasculatura cerebral, neuronal y la microglía, aumentando la permeabilidad de la barrera hematoencefálica, causa de edema y alteraciones en la función neuronal. El síndrome de respuesta inflamatoria sistémica (SIRS) puede generar fiebre, resistencia vascular sistémica baja, hipotensión arterial y gasto cardiaco elevado.

La utilización de oxigenadores de membrana y filtros en la línea arterial, así como el uso de circuitos de menor tamaño recubiertos con heparina, intentan disminuir estas complicaciones ${ }^{(19)}$. Por otra parte, es importante el control del estado ácido-base $\left(\mathrm{pH}\right.$, regulación del $\mathrm{CO}_{2}$ y saturación de oxígeno) intentando obtener una optimización de la relación FSC y el metabolismo en condiciones de hipotermia.

Mantener la glucemia dentro de valores aceptables $(140 \mathrm{mg} / \mathrm{dl})$ durante el perioperatorio, incluso 
en pacientes no diabéticos, es otro aspecto a destacar dado que el inadecuado control aumenta el daño neurológico y favorece infecciones, aumentando la mortalidad en general.

Debe evitarse que el hematocrito sea $<20 \%$, situación vinculada a mayor riesgo de stroke $e^{(13)}$. El hematocrito por debajo de $20 \%$ aumenta un $10 \%$ la incidencia de stroke, siendo su descenso por debajo del $22 \%$ un factor de riesgo independiente de $\operatorname{IEA}^{(13,32)}$.

Otro aspecto a considerar es el descenso de la presión de perfusión encefálica asociado a IEA, requiriéndose mantener estabilidad hemodinámica durante el intraoperatorio. Los mecanismos de autorregulación del FSC pueden alterarse con la CEC; descensos de la PAM pueden causar hipoperfusión e isquemia cerebral y ascensos de la misma pueden favorecer edema cerebral con aumento de la presión intracraneana. Debe realizarse monitoreo estricto de la temperatura durante la cirugía a fin de evitar hipertermia cerebral. La fase de recalentamiento es una situación que favorece la perdida de autorregulación de hasta un $20 \%$. En la cirugía cardíaca $(0,07 \%)$ y de aorta torácica $(4 \%-12 \%)$ se puede asistir a lesión en la médula espinal que resulta en paraparesia o paraplejia, siendo el mecanismo principal la embolia o la hipoperfusión medular ${ }^{(42)}$.

\section{Prevención posquirúrgica}

Es importante mantener adecuada adaptación a la $\mathrm{ARM}$, con buena ventilación y oxigenación. Mantener una adecuada presión de perfusión cerebral (PPC) es prioritario en el POCC, y dado que la PPC deriva de la diferencia entre la PAM y la presión intracraneana, se debe intentar mantener la PAM por encima de $70 \mathrm{mmHg}$ y la PAS mayor a $110 \mathrm{mmHg}$. A pesar de que no se monitoriza la presión intracraneana en el POCC, medidas como la elevación del tronco y la cabeza a $30^{\circ}$ y el adecuado equilibrio en el intercambio gaseoso favorecen la $\operatorname{PPC}^{(25,26)}$.

El control metabólico e hidroelectrolítico estricto, evitando disionías causantes de arritmias y alteraciones del medio interno es otro componente esencial del POCC. El uso de ácido acetilsalicílico (AAS) en el perioperatorio disminuye la incidencia de IEA. Se recomienda su utilización en el preoperatorio y su reinstalación a las $6 \mathrm{~h}$ del POCC si no existen contraindicaciones ${ }^{(10,11)}$. El uso concomitante de estatinas asociaría un efecto estabilizador de la placa y neuroprotector ${ }^{(7)}$. Los betabloqueantes en el período posoperatorio disminuyen la incidencia de FA. La prevención de FA aguda es importante no solo porque su instalación deteriora el gasto cardíaco sino porque se ha demostrado que aumenta la incidencia de stroke, prolonga la internación indepen- dientemente de la edad o patología previa del paciente.

Los pacientes con IEA en coma (20\% de los pacientes en coma tienen actividad eléctrica epileptógena no convulsiva) así como los pacientes que presentan mioclonías o convulsiones deben ser evaluados con electroencefalograma e ionograma completo con calcemia y magnesemia, ya que su descenso predispone a convulsiones.

La terapéutica y pronóstico del stroke en la UCI ha variado notablemente en los últimos años con la utilización de trombolíticos y trombectomía. La trombólisis sistémica está contraindicada en el POCC; a pesar de ello hay publicaciones que la plantean como conducta terapéutica en los POCC complicados con stroke $e^{(32,36-38)}$. En nuestro medio no hay experiencia .

\section{Manejo del dolor, agitación y delirio}

Las drogas analgésicas de primera elección en el POCC son los analgésicos mayores como los opioides, morfina, fentanil o remifentanil. El uso de benzodiacepinas puede aumentar la incidencia de delirio. Los analgésicos antiinflamatorios no esteroideos (AINE) como el ketoprofeno son una buena opción terapéutica, debiendo evitarse en pacientes con compromiso de la función renal.

El manejo de la agitación y delirio en el POCC requieren sedación con mínima depresión neuropsíquica. La dexmedetomidina es el fármaco de primera elección en el paciente excitado y confuso, disminuyendo la incidencia y duración del delirio con efecto sedante-analgésico titulable según la respuesta del paciente. Es una droga alfa 2 simpaticolítica que disminuye la secreción de adrenalina y noradrenalina y desciende la liberación de citoquinas, generando como consecuencia nefroprotección. Su utilización se asocia a disminución de la mortalidad global en cirugía cardíaca ${ }^{(15,16,40)}$. La dosis de mantenimiento es de 0,2 a 0,6 $\mu \mathrm{g} / \mathrm{kg} / \mathrm{h}$. El comienzo de acción es a los 15 minutos, el pico de su efecto es a la hora, y suspendida la infusión el efecto desparece a las tres horas. Puede producir bradicardia e hipotensión, por lo que debe utilizarse con precaución en pacientes en POCC valvular donde la incidencia de trastornos de la conducción es mayor.

El haloperidol es una opción terapéutica en la excitación/delirio; dosis habituales de 25 a 35 mg/día obtienen adecuado efecto terapéutico. En crisis de excitación psicomotriz se comienza con bolos 2,5 -5 mg IV repitiendo dosis según respuesta terapéutica. Se pueden utilizar hasta $5-10 \mathrm{mg} / \mathrm{h} \mathrm{IV}$.

Los antipsicóticos aumentan el intervalo QT, por lo que se recomienda precaución o suspensión 


\begin{tabular}{|c|c|c|c|}
\hline Fármaco & Dosis de carga & Dosis de mantenimiento & Efectos adversos \\
\hline Lorazepam & $\begin{array}{l}0,1 \mathrm{mg} / \mathrm{kg} \text { IV (hasta } 4 \\
\text { dosis) }\end{array}$ & $\mathrm{r} 1-5 \mathrm{mg} / \mathrm{h}$ & $\begin{array}{l}\text { Hipotensión } \\
\text { Depresión respiratoria }\end{array}$ \\
\hline Diazepam (Valium) & $0,15 \mathrm{mg} / \mathrm{kg}$ IV & & $\begin{array}{l}\text { Hipotensión } \\
\text { Depresión respiratoria }\end{array}$ \\
\hline Fenitoína & $20 \mathrm{mg} / \mathrm{kg} \mathrm{IV}$ & $\begin{array}{l}5-7 \mathrm{mg} / \mathrm{kg} / \text { día en dos a tres dosis } \\
\text { divididas }\end{array}$ & $\begin{array}{l}\text { Hipotensión } \\
\text { Bradicardia }\end{array}$ \\
\hline Tiopental & $2-5 \mathrm{mg} / \mathrm{kg}$ & $1-5 \mathrm{mg} / \mathrm{kg} / \mathrm{h}$ & $\begin{array}{l}\text { Hipotensión } \\
\text { Hipotermia } \\
\text { Depresión respiratoria }\end{array}$ \\
\hline Valproato de sodio) & $20-40 \mathrm{mg} / \mathrm{kg}$ IV & 10-15 mg/kg/día & $\begin{array}{l}\text { Encefalopatía } \\
\text { hiperamonemiante } \\
\text { Pancreatitis, } \\
\text { hepatotoxicidad, } \\
\text { trombocitopenia }\end{array}$ \\
\hline Fenobarbital & $20 \mathrm{mg} / \mathrm{kg}$ IV & $1-3 \mathrm{mg} / \mathrm{kg} /$ día en dos a tres dosis & $\begin{array}{l}\text { Hipotensión, depresión } \\
\text { respiratoria. }\end{array}$ \\
\hline Lacosamida & $200-400 \mathrm{mg}$ IV & 200-300 mg IV cada $12 \mathrm{~h}$ & $\begin{array}{l}\text { Náuseas, vómitos, trastornos } \\
\text { visuales, arritmias }\end{array}$ \\
\hline Levetiracetam & $1.000-3.000 \mathrm{mg} \mathrm{IV}$ & $2.000-4.000 \mathrm{mg} /$ día en dos dosis & Psicosis, somnolencia \\
\hline Topiramato & $\begin{array}{l}200-400 \mathrm{mg} \\
\text { NG/PO }\end{array}$ & $\begin{array}{l}\text { 300-1.600 mg/día } \\
\text { NG / PO en dos dosis divididas }\end{array}$ & $\begin{array}{l}\text { Acidosis metabólica } \\
\text { hiperclorémica }\end{array}$ \\
\hline Midazolam & $0,2 \mathrm{mg} / \mathrm{kg}$ IV & $0,1-0,4 \mathrm{mg} / \mathrm{kg} / \mathrm{h}$ & $\begin{array}{l}\text { Hipotensión, depresión } \\
\text { respiratoria. }\end{array}$ \\
\hline Propofol 1-2 mg/kg & $1,5-4 \mathrm{mg} / \mathrm{kg} / \mathrm{h}$ & $\begin{array}{l}\text { Hipotensión, } \\
\text { hipertrilgiceridemia, síndrome } \\
\text { propofol }\end{array}$ & \\
\hline Ketamina $1 \mathrm{mg} / \mathrm{kg}$ & $1-10 \mathrm{mg} / \mathrm{kg} / \mathrm{h}$ & $\begin{array}{l}\text { Depresión respiratoria, } \\
\text { Hipertensión, taquiarritmia }\end{array}$ & \\
\hline
\end{tabular}

en pacientes con QT largo. En pacientes alcoholistas la utilización de tiaprida, antipsicótico con escaso efecto extrapiramidal, se considera una opción terapéutica eficaz.

La dosis es de $100 \mathrm{mg}$ en bolo IV cada $4 \mathrm{~h}$ siendo la dosis máxima aceptada de $1.800 \mathrm{mg} /$ día.

Los antipsicóticos por vía enteral (risperidona, olanzepina, quetiapina) se utilizan cuando la excitación psicomotriz lo permite. De los antipsicóticos enterales, se recomienda quetiapina, droga de excelente absorción enteral, escaso efecto extrapiramidal con menor efecto pro-arrítmico. La dosis de inicio aconsejada es de $50 \mathrm{mg}$ v/o cada $12 \mathrm{~h}$, con dosis máxima de 1 g/día ${ }^{(16,17)}$.

\section{Manejo de las convulsiones}

Las crisis convulsivas son otra manifestación de isquemia cerebral que puede evolucionar a estado de mal epiléptico en los casos de hipoxia/hipoperfusión o alteraciones metabólicas graves persistentes. Son factores predisponentes las alteraciones cerebro- vasculares previas o la supresión de medicación anticonvulsivante que el paciente recibía previamente a la cirugía cardíaca. La mayoría de las drogas anticonvulsivantes presentan efectos sedantes con depresión respiratoria y compromiso hemodinámico. La droga de primera elección en el tratamiento de las convulsiones es el diazepam (benzodiacepina que disminuye la excitación neuronal, ansiolítico, sedante, relajante muscular e hipnótico). La dosis carga es de $0,15 \mathrm{mg} / \mathrm{kg}$ IV, con bolos posteriores de $10 \mathrm{mg} /$ dosis El tratamiento de las convulsiones en el POCC habitualmente requiere la utilización de dos drogas anticonvulsivantes, y debe realizarse control electroencefalográfico seriado.

\section{Drogas anticonvulsivantes recomendadas (tabla 3)}

Difenilhidantoína (DFH): dosis carga 15-20 $\mathrm{mg} / \mathrm{kg}$, continuando con dosis de mantenimiento de $5-7 \mathrm{mg} / \mathrm{kg} /$ día (en 3 dosis). Vida media de 24 h. El rango terapéutico es entre $10-20 \mu \mathrm{g} / \mathrm{ml}$. 


\begin{tabular}{|c|c|c|c|}
\hline & Indicaciones & Ventajas & Desventajas \\
\hline Propofol & $\begin{array}{l}\text { Sedante de primera línea } \\
\text { para tratar la HTE. } \\
\text { Sedante de primera } \\
\text { elección para el estado } \\
\text { epiléptico que no responde } \\
\text { a los anticonvulsivantes. } \\
\text { Despertar excitado post } \\
\text { PCR. }\end{array}$ & $\begin{array}{l}\text { Perfil farmacocinético. } \\
\text { Anticonvulsivante. } \\
\text { Dosis dependiente en la } \\
\text { disminución de la PIC y } \\
\text { CBF. }\end{array}$ & $\begin{array}{l}\text { Inestabilidad HD ya que } \\
\text { disminuye PAM y PPC. } \\
\text { Riesgo de síndrome de } \\
\text { infusión de propofol. } \\
\text { Pobre efecto analgésico. } \\
\text { Hipertrigliceridemia. }\end{array}$ \\
\hline Midazolam & $\begin{array}{l}\text { Sedante de segunda línea } \\
\text { para agregar cuando otros } \\
\text { sedantes son insuficientes } \\
\text { a dosis máximas. } \\
\text { Sedación en pacientes con } \\
\text { HD inestable. }\end{array}$ & $\begin{array}{l}\text { Anticonvulsivante. } \\
\text { Efecto amnésico. } \\
\text { Mayor estabilidad HD } \\
\text { comparada con el propofol. }\end{array}$ & $\begin{array}{l}\text { Riesgo de acumulación. } \\
\text { Aumenta estadía en UCI. } \\
\text { Aumenta requerimiento de } \\
\text { ARM. Aumenta riesgo de } \\
\text { delirio y stress post } \\
\text { trauma. Sin efecto } \\
\text { analgésico. }\end{array}$ \\
\hline Barbitúricos & $\begin{array}{l}\text { Terapia de rescate en la } \\
\text { HTE y en el estado } \\
\text { epiléptico refractario y } \\
\text { superrefractario. }\end{array}$ & $\begin{array}{l}\text { Fuerte efecto en la } \\
\text { disminución de la PIC. } \\
\text { Supresión en el EEG. }\end{array}$ & $\begin{array}{l}\text { Hipotensión, baja PAM y } \\
\text { PPC. } \\
\text { Disfunción suprarrenal. } \\
\text { Inmunosupresión. } \\
\text { Nefro y Hepatotoxicidad. } \\
\text { Vida media larga. }\end{array}$ \\
\hline Opioides & $\begin{array}{l}\text { Analgesia. } \\
\text { Adaptación a la ARM. }\end{array}$ & & $\begin{array}{l}\text { Pobre efecto sobre la PIC. } \\
\text { Riesgo de acumulación } \\
\text { excepto con remifentanilo. } \\
\text { Remifentanilo: } \\
\text { dependencia en } \\
\text { hiperalgesia. }\end{array}$ \\
\hline Dexmedetomidina & $\begin{array}{l}\text { Sedación leve, destete o } \\
\text { asociación en casos de } \\
\text { agitación con otros } \\
\text { sedantes. }\end{array}$ & $\begin{array}{l}\text { Efecto analgésico, sedante } \\
\text { y ansiolítico. } \\
\text { De corta acción y sin } \\
\text { acumulación. } \\
\text { Mínima depresión } \\
\text { respiratoria. Bajo riesgo de } \\
\text { delirio. }\end{array}$ & $\begin{array}{l}\text { Bradicardia e hipotensión. } \\
\text { Sedación profunda. } \\
\text { Costoso }\end{array}$ \\
\hline Ketamina & $\begin{array}{l}\text { Control de los estados } \\
\text { epilépticos refractarios o } \\
\text { superrefractarios cuando } \\
\text { los agentes GABA no son } \\
\text { efectivos. }\end{array}$ & $\begin{array}{l}\text { Analgésico y efecto } \\
\text { sedante. } \\
\text { Hemodinámicamente } \\
\text { estable. } \\
\text { Tratamiento del estado } \\
\text { epiléptico refractario. } \\
\text { Rápido inicio y cese de } \\
\text { acción. } \\
\text { No depresión respiratoria. }\end{array}$ & $\begin{array}{l}\text { Probablemente sin efecto } \\
\text { sobre la PIC en pacientes } \\
\text { sedados y ventilados. } \\
\text { Alucinaciones. } \\
\text { Colestasis, inflamación de } \\
\text { la mucosa urinaria. }\end{array}$ \\
\hline
\end{tabular}

Levetiracetam: disponible por vía enteral y parenteral. Dosis carga de $10 \mathrm{mg} / \mathrm{kg}$ IV y dosis de mantenimiento de 10-20 mg/kg/día. Dosis máxima 3-4 g/día (administradas cada $12 \mathrm{~h}$ ), dosis mayores no han evidenciado efectos significativos como anticonvulsivante. La absorción enteral es excelente, con una biodisponibilidad de aproximadamente $100 \%$. El rango terapéutico en sangre es de $12-46 \mu \mathrm{g} / \mathrm{ml}$. Se asocia a menor incidencia de plaquetopenia en comparación con otros anticonvulsivantes. Si la situación clínica lo requiere puede asociarse a $\mathrm{DFH}^{(27,28)}$.

Valproato sódico: anticonvulsivante; puede utilizarse por vía parenteral y enteral. Dosis carga de $20-40 \mathrm{mg} / \mathrm{kg}$ IV y dosis de mantenimiento de 10-15 mg/kg/día. Rango terapéutico de 50-100 $\mu \mathrm{g} / \mathrm{ml}$. 
Si el paciente continúa convulsando y evoluciona a estado de mal convulsivo (tabla 4):

Propofol: anestésico de rápida acción (30 segundos) y corta duración; anticonvulsivante y altamente efectivo en la reducción de la presión intracraneana; asocia efecto broncodilatador.

El contenido de cada ampolla es de $10 \mathrm{mg} / \mathrm{ml}$. Dosis carga de $1-2 \mathrm{mg} / \mathrm{kg} /$ dosis con dosis de mantenimiento de $1,5-4 \mathrm{mg} / \mathrm{kg} / \mathrm{h}$, ajustando la misma según respuesta. Suspendida su infusión la recuperación se obtiene entre 7-10 min.

Midazolam: benzodiacepina con efecto anticonvulsivante, sedante e hipnótico. Produce menor compromiso hemodinámico que el propofol. Dosis carga de $0,2 \mathrm{mg} / \mathrm{kg}$ IV seguido por infusión de $0,1-0,4 \mathrm{mg} / \mathrm{kg} / \mathrm{h}$.

Ketamina: droga anestésica con efecto broncodilatador, útil en pacientes con inestabilidad hemodinámica. Dosis carga de $1 \mathrm{mg} / \mathrm{kg}$ IV y dosis de mantenimiento de $1-10 \mathrm{mg} / \mathrm{kg} / \mathrm{h}$.

Tiopental: barbitúrico; produce compromiso hemodinámico (hipovolemia, hipotensión, gasto bajo), hipotermia e inmunosupresión. Dosis carga de 3-5 $\mathrm{mg} / \mathrm{kg}$ IV y dosis de mantenimiento entre 1 y 5 $\mathrm{mg} / \mathrm{kg} / \mathrm{h}$.

\section{Conclusiones}

Las complicaciones neurológicas, que suelen provocar secuelas invalidantes permanentes, tienen un impacto negativo en la sobrevida y la calidad de vida de quienes van a cirugía cardíaca. Las claves para minimizar este daño incluyen el reconocimiento de los factores predisponentes y el diagnóstico precoz de las complicaciones, con el objetivo de implementar estrategias terapéuticas eficientes. Este grupo heterogéneo de complicaciones, no sólo afecta la sobrevida, sino que también genera otras complicaciones como asistencia respiratoria prlongada con sus consecuencias negativas, dehiscencia esternal que favorece las infecciones del sitio quirúrgico, e internaciones prolongadas con consecuencias directas en los costos sanitarios.

Omar Montes de Oca,

https://orcid.org/0000-0001-9317-9529

Mauricio Saez, https://orcid.org/0000-0003-4905-0837

Este artículo fue aceptado para su publicación por: Editora adjunta Dra. Yamel Ache

\section{Bibliografía}

1. Seco M, Edelman J, Van Boxtel B, Forrest P, Byrom M, Wilson M, et al. Neurologic injury and protection in adult cardiac and aortic surgery. J Cardiothorac Vasc Anesth. 2015; 29(1):185-95. doi: 10.1053/j.jvca.2014.07.026

2. Sousa-Uva M, Neumann F, Ahlsson A, Alfonso F, Banning A, Benedetto U, et al; ESC Scientific Document Group. 2018 ESC/EACTS Guidelines on myocardial revascularization. Eur J Cardiothorac Surg. 2019; 55(1):4-90. doi: 10.1093/ ejcts/ezy289

3. Dabrowski W, Rzecki Z, Pilat J, Czajkowski M. Brain damage in cardiac surgery patients. Curr Opin Pharmacol. 2012; 12(2):189-94. doi: 10.1016/j.coph. 2012.01.013

4. Newman M, Grocott H, Mathew J, White W, Landolfo K, Reves J, et al; Neurologic Outcome Research Group and the Cardiothoracic Anesthesia Research Endeavors (CARE) Investigators of the Duke Heart Center. Report of the substudy assessing the impact of neurocognitive function on quality of life 5 years after cardiac surgery. Stroke. 2001; 32(12):2874-81. doi: 10.1161/hs 1201.099803

5. Ramlawi B, Rudolph J, Mieno S, Khabbaz K, Sodha N, Boodhwani M, et al. Serologic markers of brain injury and cognitive function after cardiopulmonary bypass. Ann Surg. 2006; 244(4):593-601. doi: 10.1097/01.sla.0000239087.00826.b4

6. Ben-Abraham R, Weinbroum A, Dekel B, Paret G. Chemokines and the inflammatory response following cardiopulmonary bypass - a new target for therapeutic intervention?-A review. Paediatr Anaesth. 2003; 13(8):655-61. doi: 10.1046/j.1460-9592.2003. 01069.x

7. Bouchard D, Carrier M, Demers P, Cartier R, Pellerin M, Perrault L, et al. Statin in combination with â-blocker therapy reduces postoperative stroke after coronary artery bypass graft surgery. Ann Thorac Surg. 2011; 91(3):654-9. doi: 10.1016/j.at horacsur.2010.11.036

8. McKhann G, Grega M, Borowicz LJr, Baumgartner W, Selnes O. Stroke and encephalopathy after cardiac surgery: an update. Stroke. 2006; 37(2):562-71. doi: 10.1161/01.STR.0000199032.78782.6c

9. Lee V, Wijdicks E. Neurologic complications of cardiac surgery. Continuum (Minneap Minn) 2008; 14(1):145-64. doi: 10.1212/01.CON.0000299990. 24695.06

10. Mangano D; Multicenter Study of Perioperative Ischemia Research Group. Aspirin and mortality from coronary bypass surgery. N Engl J Med. 2002; 347(17):1309-17. doi: 10.1056/NEJMoa020798 
11. Bybee K, Powell B, Valeti U, Rosales A, Kopecky $\mathbf{S}$, Mullany $\mathbf{C}$, et al. Preoperative aspirin therapy is associated with improved postoperative outcomes in patients undergoing coronary artery bypass grafting. Circulation. 2005; $112(9$ Suppl):I286-92. doi: 10.1161/CIRCULATIONAHA. 104.522805

12. Durand D, Perler B, Roseborough G, Grega M, Borowicz LJr, Baumgartner W, et al. Mandatory versus selective preoperative carotid screening: a retrospective analysis. Ann Thorac Surg. 2004 78(1):159-66. doi: 10.1016/j.athoracsur.2004.02.024

13. Karkouti K, Djaiani G, Borger M, Beattie W, Fedorko L, Wijeysundera D, et al. Low hematocrit during cardiopulmonary bypass is associated with increased risk of perioperative stroke in cardiac surgery. Ann Thorac Surg. 2005; 80(4):1381-7. doi: 10.1016/j.athoracsur.2005.03.137

14. Reade M, Finfer S. Sedation and delirium in the in tensive care unit. N Engl J Med. 2014; 370(5):444-54. doi: 10.1056/NEJMra1208705

15. Ji F, Li Z, Nguyen H, Young N, Shi P, Fleming $\mathbf{N}$, et al. Perioperative dexmedetomidine improves outcomes of cardiac surgery. Circulation. 2013; 127(15):1576-84. doi: 10.1161/CIRCULATIONAHA 112.000936

16. Barr J, Fraser G, Puntillo K, Ely E, Gélinas C, Dasta J, et al; American College of Critical Care Medicine. Clinical practice guidelines for the management of pain, agitation, and delirium in adult patients in the intensive care unit. Crit Care Med. 2013; 41(1):263-306. doi: 10.1097/CCM.0b013e $3182783 b 72$

17. Selnes O, Gottesman R, Grega M, Baumgartner W, Zeger S, McKhann G. Cognitive and neurologic outcomes after coronary-artery bypass surgery. N Engl J Med. 2012; 366(3):250-7. doi: 10.1056/NEJ Mra1100109

18. Baufreton C. Role of surgical factors in strokes after cardiac surgery. Arch Cardiovasc Dis. 2010; 103(5):326-32. doi: 10.1016/j.acvd.2009.12.007

19. Lee E, Choi K, Ryu J, Jeon S, Lee S, Park S, et al. Stroke risk after coronary artery bypass graft surgery and extent of cerebral artery atherosclerosis. J Am Coll Cardiol. 2011; 57(18):1811-8. doi: 10.1016/j.jacc.2010.12.026

20. Grocott H, Yoshitani K. Neuroprotection during cardiac surgery. J Anesth. 2007; 21(3):367-77. doi: 10.1007/s00540-007-0514-1

21. Allen J, Alexander E. Prevention, recognition, and management of delirium in the intensive care unit. AACN Adv Crit Care. 2012; 23(1):5-11. doi: 10.1097/NCI.0b013e31822c3633

22. Windecker S, Kolh P, Alfonso F, Collet J, Cremer J, Falk V, et al. 2014 ESC/EACTS Guidelines on myocardial revascularization: The Task Force on Myocardial Revascularization of the European Society of Cardiology (ESC) and the European Association for Cardio-Thoracic Surgery (EACTS)Developed with the special contribution of the European Association of Percutaneous Cardiovascular Interventions (EAPCI). Eur Heart J. 2014; 35(37):2541-619. doi: 10.1093/eurheartj/ehu278

23. Hillis L, Smith P, Anderson J, Bittl J, Bridges C, Byrne J, et al. 2011 ACCF/AHA Guideline for Coronary Artery Bypass Graft Surgery: a report of the American College of Cardiology Foundation/American Heart Association Task Force on Practice Guidelines. Circulation. 2011; 124(23):e652-735. doi: 10.1161/CIR.0b013e31823c074e

24. Sandsmark D, Sheth K. Management of increased intracranial pressure. Curr Treat Options Neurol. 2014; 16(2):272. doi: 10.1007/s11940-013-0272-3

25. Latorre J, Greer D. Management of acute intracranial hypertension: a review. Neurologist. 2009; 15(4):193-207. doi: 10.1097/NRL.0b013e31819f956a

26. Sultan I, Bianco V, Kilic A, Jovin T, Jadhav A, Jankowitz B, et al. Predictors and Outcomes of Ischemic Stroke After Cardiac Surgery. Ann Thorac Surg. 2020; 110(2):448-56. doi: 10.1016/j.athoracsur.2020.02.025

27. Chen C, Chen T, Tu P, Wu V, Yang C, Wang A, et al. Long-Term Outcomes for Patients With Stroke After Coronary and Valve Surgery. Ann Thorac Surg. 2018; 106(1):85-91. doi: 10.1016/j.athoracsur.2018.01.067

28. Patel B, Reinert N, Al-Robaidi K, Gao X, Fabio A, Esper S, et al. Independent Predictors of Perioperative Stroke-Related Mortality after Cardiac Surgery. J Stroke Cerebrovasc Dis. 2020; 29(5):104711. doi: 10.1016/j.jstrokecerebrovasdis.2020.104711

29. Yuan S, Lin H. Postoperative Cognitive Dysfunction after Coronary Artery Bypass Grafting. Braz J Cardiovasc Surg. 2019; 34(1):76-84. doi: 10.21470/1678-9741-2018-0165

30. Mehta A, Choxi R, Gleason T, Wechsler L, Jovin T, Thirumala P. Carotid Artery Disease as a Predictor of In-Hospital Postoperative Stroke After Coronary Artery Bypass Grafting From 1999 to 2011. J Cardiothorac Vasc Anesth. 2018; 32(4):1587-96. doi: 10.1053/j.jvca.2017.10.010.

31. Kashani H, Mosienko L, Grocott B, Glezerson B, Grocott H. Postcardiac Surgery Acute Stroke Therapies: A Systematic Review. J Cardiothorac Vasc Anesth. 2020; 34(9):2349-54. doi: 10.1053/j.jvca.2020.03.041

32. Evans A, Weiner M, Arora R, Chung I, Deshpande $\mathbf{R}$, Varghese $\mathbf{R}$, et al. Current approach to diagnosis and treatment of delirium after cardiac surgery. Ann Card Anaesth. 2016; 19(2):328-37. doi: 10.4103/0971-9784.179634 
33. Farrokh S, Tahsili-Fahadan P, Ritzl E, Lewin J3rd, Mirski M. Antiepileptic drugs in critically ill patients. Crit Care. 2018; 22(1):153. doi: 10.1186/s13054-018-2066-1

34. Sociedad Europea de Cardiologia. European Association for Cardio-Thoracic Surgery. Grupo de Trabajo sobre revascularización miocárdica. Guía ESC/EACTS 2018 sobre revascularización miocárdica. Rev Esp Cardiol. 2019; 72(1):73.e1-76. doi: 10.1016/j.rec.2018.11.012

35. Hillis L, Smith P, Anderson J, Bittl J, Bridges C, Byrne J, et al. 2011 ACCF/AHA Guideline for coronary artery bypass graft surgery: executive summary: a report of the American College of Cardiology Foundation/American Heart Association Task Force on Practice Guidelines. Circulation. 2011; 124(23):2610-42. doi: 10.1161/CIR.0b013e31823b5fee

36. Moazami N, Smedira N, McCarthy P, Katzan I, Sila C, Lytle B, et al. Safety and efficacy of intraarterial thrombolysis for perioperative stroke after cardiac operation. Ann Thorac Surg. 2001; 72(6):1933-7. doi: 10.1016/s0003-4975(01)03030-2

37. Al-Khawaldeh M, Haddad O, Alshabatat A, Deo S, Rababa'h A, Altarabsheh S. A Successful Endovascular Treatment of an Ischemic Stroke follo- wing Cardiac Surgery. Oman Med J. 2015; 30(6):473-6. doi: 10.5001/omj.2015.92

38. Haider A, Garg P, Watson I, Leonard D, Khan U, Haque A, et al. Mechanical Thrombectomy for Acute Ischemic Stroke After Cardiac Surgery. Cureus. 2017; 9(4):e1150. doi: 10.7759/cureus. 1150

39. Wu M, Liang Y, Dai Z, Wang S. Perioperative dexmedetomidine reduces delirium after cardiac surgery: A meta-analysis of randomized controlled trials. J Clin Anesth. 2018; 50:33-42. doi: 10.1016/ j.jclinane.2018.06.045

40. Fernández Domínguez J, Martínez Sánchez P, García Rodríguez R, Mateos Marcos V, Valdueza J. El eco-doppler color transcraneal en el estudio vascular cerebral. Neurol Arg. 2012; 4(3):132-43.

41. Kargiotis O, Psychogios K, Safouris A, Magoufis G, Zervas P, Stamboulis E, et al. The Role of transcranial doppler monitoring in patients with multi-territory acute embolic strokes: a review. J Neuroimaging. 2019; 29(3):309-22. doi: 10.1111/jon. 12602

42. Irons S, Hoffman J. Functional outcomes of a patient with acute paraplegia after open heart surgery: a case report. Cardiopulm Phys Ther J. 2016; 27(4):115-21. 